Check for updates

Cite this: RSC Adv., 2018, 8, 32454

Received 6th June 2018

Accepted 12th September 2018

DOI: $10.1039 / \mathrm{c} 8 \mathrm{ra04819c}$

rsc.li/rsc-advances

\section{Development of a fluorescence assay for highly sensitive detection of Pseudomonas aeruginosa based on an aptamer-carbon dots/graphene oxide system $\dagger$}

\begin{abstract}
Hongying Wang, $\xi^{a}$ Zhe Chi, (D) $t^{* a}$ Ying Cong, ${ }^{a}$ Zhuangzhuang Wang, ${ }^{a}$ Fei Jiang, ${ }^{a}$ Jiayue Geng, ${ }^{a}$ Peng Zhang, ${ }^{* b}$ Peng Ju, ${ }^{c}$ Quanjiang Dong ${ }^{d}$ and Chenguang Liu*a

An aptamer-based fluorescence assay for culture-independent detection of Pseudomonas aeruginosa was developed. This assay was enabled by highly specific aptamers conjugated with photoluminescent carbon dots (CDs) as the fluorescent probe and graphene oxide $(G O)$ as the quencher. Specially, high-throughput sequencing was achieved during systematic evolution of ligands via exponential enrichment (SELEX) for accurate recognition of aptamers. This assay displayed high specificity towards $P$. aeruginosa and was resistant to interference by other ubiquitous bacteria including Escherichia coli, Bacillus subtilis, Staphylococcus aureus, Enterococcus faecalis, and Clostridium perfringens. After the conditions were optimized, this assay achieved a wide detection range for $P$. aeruginosa varying from $10^{1} \mathrm{CFU} \mathrm{mL}^{-1}$ to

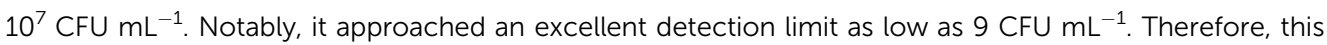
fluorescence assay was considered successfully developed for highly sensitive detection of $P$. aeruginosa. This assay also detected the contamination of $P$. aeruginosa in tap water and commercial bottled water, thereby suggesting its potential application in real water samples.
\end{abstract}

\section{Introduction}

Pseudomonas aeruginosa is a ubiquitous opportunistic pathogen, which exists in moist environments. Its characteristics make it a significant threat to human health and it is now being monitored clinically and in the food industry. ${ }^{1}$ Although traditional methods for culture-dependent detection of $P$. aeruginosa are reliable, ${ }^{\mathbf{1 , 2}}$ more rapid and sensitive detection methods are still demanded. ${ }^{1}$ In recent years, aptamer-based biosensors and assays have displayed greater advantages in detecting pathogenic microorganisms with rapidity and high specificity and sensitivity. ${ }^{3}$

Aptamers are well known to be obtained through systematic evolution of ligands via exponential enrichment (SELEX). ${ }^{4}$

${ }^{a}$ College of Marine Life Science, Ocean University of China, Qingdao 266003, P. R. China.E-mail: cz1108@ouc.edu.cn; liucg@ouc.edu.cn

${ }^{b}$ Research Centre of Plant Functional Components, Tobacco Research Institute of Chinese Academy of Agricultural Sciences, Qingdao, 266001, P. R. China. E-mail: zhangpeng@caas.cn

'Key Laboratory of Marine Bioactive Substances and Analytical Technology, Marine Ecology Center, The First Institute of Oceanography, State Oceanic Administration (SOA), 6 Xianxialing Road, Qingdao 266061, P. R. China

${ }^{d}$ Central Laboratory and Department of Gastroenterology, Qingdao Municipal Hospital, Qingdao 266071, P. R. China

$\dagger$ Electronic supplementary information (ESI) available. See DOI: $10.1039 / \mathrm{c} 8 \mathrm{ra04819c}$

$\ddagger$ These authors contribute equally to this article.
Presently, high-throughput sequencing (HTS), which yields extensive data at the gigabyte level, can guarantee adequate sequence coverage, thereby enabling accurate recognition of the most abundant sequences in polymerase chain reaction (PCR) products in any round of SELEX; this provides a novel comprehensive method for precise identification of target aptamer. ${ }^{5}$ Therefore, HTS is incorporated into whole-bacteria SELEX (WB-SELEX) in this study.

Aptamer-based biosensors and assays employ various sensing methodologies. 6 The fluorometric assays are more promising and advantageous than colorimetric assays, owing to their increased sensitivity. ${ }^{6}$ Among numerous fluorophores, carbon dots (CDs) have received increasing attention for their excellent optical performance for enhanced sensitivity and their large-scale synthesis is easy and cost-effective. ${ }^{7,8}$ Recently, graphene oxide (GO) has been more commonly applied in fluorometric biosensors. ${ }^{6}$ GO can serve as an anchor for aptamers owing to the $\pi-\pi$ stacking interactions between them. ${ }^{9}$ Moreover, they have great fluorescence quenching capability for fluorophores owing to fluorescence resonance energy transfer (FRET), ${ }^{6,9}$ which is similar to the characteristics of nanographite, which can effectively reduce the background fluorescence signals of CDs and improve sensitivity. ${ }^{8}$ Hence, a fluorescence assay, which utilizes the aptamer-CD probe/GO system might be ideal for achieving rapid and highly sensitive detection of $P$. aeruginosa in a culture-independent manner. 


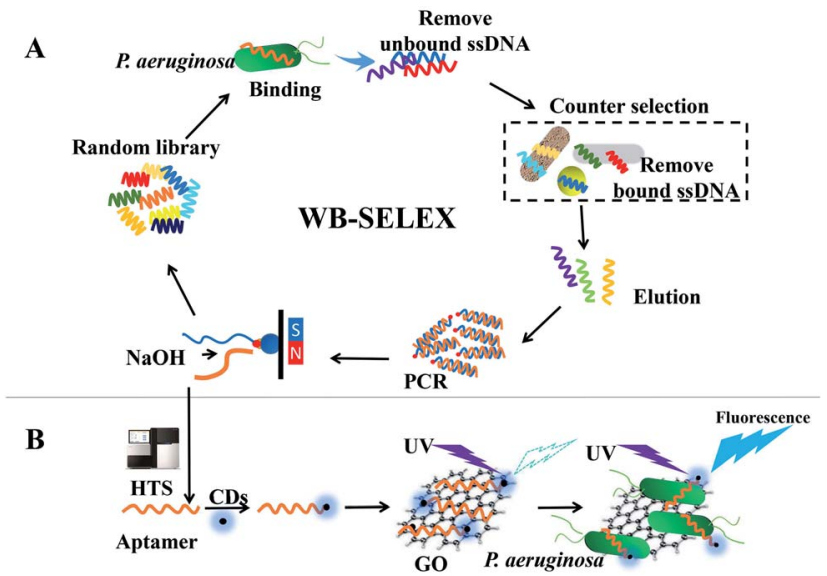

Scheme 1 A schematic representation for the development of the fluorescence assay to detect $P$. aeruginosa. (A), whole-bacteria SELEX (WB-SLEX) for selecting aptamer candidates for $P$. aeruginosa. Highthroughput sequencing (HTS) was performed for the recognition of aptamer candidates. (B), the working principle of aptamer-carbon dots (CDs)/graphene oxide (GO) system for fluorescence detection of $P$. aeruginosa. UV, ultraviolet. When there are no cells of $P$. aeruginosa, the aptamer-CDs probes are attached to the surface of $G O$ and their fluorescence is quenched by GO. While when confronting with the cells of $P$. aeruginosa, the aptamer-CDs probes are pulled away from the surface of GO and the fluorescence is recovered.

The present study aimed to develop such a fluorescence assay (Scheme 1). Five aptamer candidates for P. aeruginosa were recognized via WB-SELEX with the HTS technique. Then, these aptamer candidates were conjugated with photoluminescent CDs to synthesize three fluorescent probes. With these probes, their equilibrium dissociation constant was determined, thereby aiding the exclusion of two aptamer candidates. Subsequently, the remaining three probes were mixed with graphene oxide (GO) to form the detection systems. By performing the detection to $P$. aeruginosa and six other interfering bacteria, only one aptamer-CDs probes showed the highest specificity to $P$. aeruginosa. With this highly specific aptamer-CDs/GO system, a highly sensitive fluorescence assay for the detection of $P$. aeruginosa mediated was successfully accomplished.

\section{Experimental}

\subsection{Strains and culture conditions}

Pseudomonas aeruginosa ATCC 27853 was used for aptamer selection. Escherichia coli ATCC 25922, Bacillus subtilis ATCC 6051, and Staphylococcus aureus ATCC 12600 were used for counter selections. Pseudomonas putida ATCC 12633, Enterococcus faecalis ATCC 19433, and Clostridium perfringens ATCC 13124, along with the aforementioned counter selection bacteria were used as interfering bacteria to determine the specificities of aptamer candidates. All the aerobic bacterial strains were cultured at $37^{\circ} \mathrm{C}$ in LB medium $\left(10 \mathrm{~g} \mathrm{~L}^{-1}\right.$ tryptone, $5 \mathrm{~g} \mathrm{~L}^{-1}$ yeast extract, and $10 \mathrm{~g} \mathrm{~L}^{-1} \mathrm{NaCl} ; \mathrm{pH}$ 7.2-7.4) for $16 \mathrm{~h}$ before harvest. The anaerobic bacteria were cultured in fluid thioglycolate medium (Aikb, Qingdao) and in an anaerobic workstation (BUGBOX, Ruskinn, Bridgend, UK) for $48 \mathrm{~h}$ at $37^{\circ} \mathrm{C}$. The cells were harvested via centrifugation at $1000 \times g$ for $3 \mathrm{~min}$ and washed twice with binding buffer $(50 \mathrm{mM}$ Tris- $\mathrm{HCl}$, $100 \mathrm{mM} \mathrm{NaCl}, 5 \mathrm{mM} \mathrm{KCl}$, and $1 \mathrm{mM} \mathrm{MgCl}$; pH 7.5) before use.

\subsection{SELEX procedures}

The SELEX procedures were followed in accordance with previously described methods ${ }^{\mathbf{1 0}}$ with some improvements. Initially, the ssDNA library was synthesized by GenScript (Nanjing, China) and consisted of a central random sequence of 40 nucleotides (nt) flanked by two primer hybridization sites $\left(5^{\prime}\right.$ TGGACCTTGCGATTGCGATTGA CAGC (40 nt) GCAGACATGAGTCTCAGGAC-3'). A counter selection was implemented immediately in the first round of SELEX, ${ }^{11}$ using $E$. coli ATCC 25922, B. subtilis ATCC 6051, and S. aureus ATCC 12600 together as counter selection bacteria (final density $1 \times 10^{7} \mathrm{CFU} \mathrm{mL}^{-1}$ for each culture) in $300 \mu \mathrm{L}$ binding buffer containing $1.3 \mathrm{nmol}$ of the initial ssDNA library. The rest operations were consistent with conventional methods, and an additional 14 rounds were carried out. Selection conditions in every round are listed in Table S1†.

\subsection{Recognition of aptamer candidates via HTS}

A comprehensive study of the sequences in the ssDNA pools in the 9th, 12th, and 15th rounds of SELEX were accomplished via HTS. Briefly, $2 \mu \mathrm{g}$ of ssDNA were enriched from PCR products generated from the last round and sequenced at Novogene Co. Ltd. (Beijing, China) via the Illumina HiSeq 3000 platform for PE 150 sequencing to obtain data of 1 gigabyte. Sequences thus obtained were clustered in accordance with their identities to reveal the abundance of each sequence inside the ssDNA pool. The five most abundant ssDNAs were selected as aptamer candidates. The free energies of aptamer candidates were analyzed using the DNAMAN software ${ }^{12}$ and secondary structures of the aptamer candidates were predicted using Mfold program (http://mfold.rna.albany.edu/?q=mfold). ${ }^{13}$

\subsection{Preparation and characterization of CDs and aptamer- CDs conjugates}

CDs and aptamer-CD conjugates were synthesized using the method described previously. ${ }^{\mathbf{8} 14}$ The obtained CDs and aptamer candidate-CDs conjugates were characterized via HRTEM and agarose gel electrophoresis, ${ }^{8} \mathrm{UV}$-vis scanning, and scanning of excitation and emission spectra. ${ }^{14}$

\subsection{Determination of the equilibrium dissociation constant}

The equilibrium dissociation constant $\left(K_{\mathrm{d}}\right)$ of the five aptamer candidates were measured in accordance with a previously described method, ${ }^{10,15}$ with some modifications. Generally, $P$. aeruginos $a$ at the final density of $1 \times 10^{7} \mathrm{CFU} \mathrm{mL}^{-1}$ were incubated in a $300 \mu \mathrm{L}$ binding buffer at $37{ }^{\circ} \mathrm{C}$ with each aptamer candidate-CD probe synthesized at the different final concentrations of $0,50,100,150,200,250,300,350$, and $400 \mathrm{nM}$. The fluorescence intensity was measured at an excitation 
wavelength of $450 \mathrm{~nm}$ and the $K_{\mathrm{d}}$ values were calculated as described previously. ${ }^{\mathbf{1 0}}$

\subsection{Specificity test}

For determining the specificity of the five aptamer candidates to $P$. aeruginosa, five corresponding aptamer candidate-CDs/GO systems were constructed, which respectively consisted of $100 \mathrm{~g} \mathrm{~mL}^{-1}$ of GO (diameter 10-50 $\mu \mathrm{m}$ ) and $15 \mu \mathrm{M}$ of each aptamer-CD probe in $300 \mu \mathrm{L}$ binding buffer. These systems were incubated for $1 \mathrm{~h}$ at $37{ }^{\circ} \mathrm{C}$ and their fluorescence intensities were measured at an excitation wavelength of $450 \mathrm{~nm}$. Thereafter, $100 \mu \mathrm{L}$ of $P$. aeruginosa $\left(1 \times 10^{7} \mathrm{CFU} \mathrm{mL}^{-1}\right)$ was introduced into each system and incubated at $37^{\circ} \mathrm{C}$ for $30 \mathrm{~min}$. The fluorescence intensities were measured again at an excitation wavelength of $450 \mathrm{~nm}$. Groups not containing $P$. aeruginosa served as the negative control. The fluorescence recovery ratio was determined as follows: $\Delta I=\left[\left(F-F_{0}\right) / F_{0}\right](F=$ the fluorescence intensity of each aptamer-CDs/GO system in the presence of $P$. aeruginosa, $F_{0}=$ the fluorescence intensity in the absence of $P$. aeruginosa). To evaluate the specificities of each aptamerCD probe, fluorescence recovery ratios were determined using these five aptamer-CD/GO systems against the six different interfering bacteria, as stated above at a density of $1 \times 10^{7} \mathrm{CFU}$ $\mathrm{mL}^{-1}$. The statistical analysis was performed by Student's $t$ test, $p<0.05$ was considered to have difference and $p<0.01$ was considered to have significant difference.

\subsection{Optimization of reacting conditions}

For optimization of GO concentration, the concentration of aptamer-CD probes was fixed as $15 \mu \mathrm{M}$, as reported previously, ${ }^{8}$ whereas GO concentration was set differently as $0,25,50,75$, $100,125,150,175,200$, and $225 \mu \mathrm{g} \mathrm{mL}{ }^{-1}$, respectively. After incubation in $300 \mu \mathrm{L}$ of binding buffer ( $\mathrm{pH}$ 7.5) for $30 \mathrm{~min}$, the fluorescence intensities were determined as described above. Reaction time was optimized by measuring fluorescence

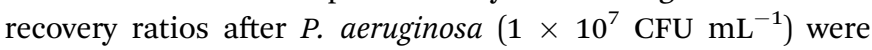
subjected to a previously optimized aptamer-CDs/GO system and incubated for $0,15,30,45,60,75,90,105,120$, and $135 \mathrm{~min}$, respectively, following the previous procedures.

\subsection{Detection range and limit for $P$. aeruginosa}

The detection range was analysed using the aptamer-CDs/GO system under optimized conditions, with $P$. aeruginosa at the following densities: $10,1 \times 10^{2}, 1 \times 10^{3}, 1 \times 10^{4}, 1 \times 10^{5}, 1 \times$ $10^{6}$, and $1 \times 10^{7} \mathrm{CFU} \mathrm{mL}^{-1}$. Fluorescence recovery ratios were measure as described above. The limit of detection was determined in accordance with a previously reported method. ${ }^{\mathbf{1 6}}$

\subsection{Detection of $P$. aeruginosa in artificially contaminated samples}

The aptamer-CDs/GO system was applied in real time with tap water and commercial mineral water (Nestle Co. Ltd., Beijing, China). They were first treated with the aptamer-CDs/GO system from this study to detect the $P$. aeruginosa in accordance with the procedures in the previous paragraphs. Thereafter, they were artificially contaminated with $P$. aeruginosa at $1 \times 10^{1}, 1 \times$ $10^{2}$, and $1 \times 10^{3} \mathrm{CFU} \mathrm{mL} \mathrm{m}^{-1}$, respectively. The contaminated samples were also subjected to the aptamer-CDs/GO system with the same procedures. Sterilized distilled water was used as a negative control.

\section{Results and discussion}

\subsection{Efficient HTS-assisted recognition of aptamer candidates}

A specific aptamer is the first element for developing the fluorescence assay proposed herein. HTS is currently known to greatly improve efficiency in analyzing sequences for sSDNA pools, which are generated in the process of SELEX and accurately recognizes highly abundant sequences as aptamer candidates., ${ }^{517}$ In this study, HTS revealed that PA1, PA2, PA3, PA4, and PA5 (Table 1) were the most abundant sequences in the SSDNA pools from the $9^{\text {th }}, 12^{\text {th }}$, and $15^{\text {th }}$ round of SELEX (Fig. S1†); these five ssDNA sequences were selected as aptamer candidates. Moreover, they all approached their maximum abundances at the $12^{\text {th }}$ round (Fig. S1 $\dagger$ ), indicating that twelve rounds of SELEX were most appropriate.

HTS in this study yielded approximately $1.7 \times 10^{6}$ strands for each ssDNA pool above and the repeats of the five aptamers were in tens of thousands (Fig. S1 $\dagger$ ). Nevertheless, in the SELEX procedure for Listeria species, only fifty clones were sequenced and only one or two repeats were detected within these fifty clones. ${ }^{15}$ This rendered the selected aptamer not representative but rather stochastic because of high varieties within more than a million of sequences in the ssDNA pools. ${ }^{5}$ Hence, the use of HTS in SELEX greatly assisted the recognition of aptamer candidates with high specificity and efficiency.

Table 1 shows that these five candidates exhibited a much lower free energy $(\Delta G)$ than previously reported aptamers for $P$. aeruginosa ${ }^{\mathbf{1 0}}$ indicating a greater ease of formatting stable secondary structures. Moreover, the secondary structures shown in Fig. $\mathrm{S} 2 \dagger$ demonstrated that the aptamer candidates identified via HTS could form more and complicated stem-loop branches around relatively small central loops, which might enable their accommodation at $P$. aeruginosa cell walls with increased specificity. Subsequently, all aptamer candidates obtained via HTS were assessed for their $K_{\mathrm{d}}$ values.

\section{2 $K_{\mathrm{d}}$ values for aptamer candidates}

To determine the $K_{\mathrm{d}}$ value of each aptamer candidate, CDs were prepared via the hydrothermal method with citric acid and ethylenediamine, as reported previously. ${ }^{\mathbf{1 4}}$ Characterization of CDs synthesized herein is illustrated in Fig. S3†. The results (Fig. S3-A and $\mathrm{B}^{\dagger}$ ) are consistent with previous findings, ${ }^{\mathbf{8 , 1 4}}$ indicating that the CDs were successfully prepared and could be used as fluorophores.

Subsequently, the obtained CDs were conjugated to aminemodified five aptamer candidates the via amide bond catalyzed by EDC and NHS. ${ }^{8}$ After conjugation, they were subjected to agarose gel electrophoresis. Fig. S3-C $\dagger$ shows that the CDconjugated ssDNA (lanes 1-5) of aptamer candidates migrated 
slower than their unconjugated counterparts (lanes 6-10), owing to an increased mass-to-charge ratio because of ssDNACDs conjugation, ${ }^{8}$ indicating that the covalent bond between the CDs and aptamer candidates was successfully formed. Thereafter, the fluorescence emission spectra of the aptamerCDs nanoconjugate was analysed again. Notably, a redshift of the fluorescence emission peak was observed for the ssDNACDs conjugates as compared with original CDs (Fig. S3-D†, the PA1-CDs was shown as an example, the rest four aptamerCDs had the same results). Because the aptamers and CDs were covalently conjugated herein, it introduced numerous $\mathrm{N}$ atoms to $\mathrm{CD}$ surface. The introduction of $\mathrm{N}$ atoms to the $\mathrm{CD}$ surface would lead to a redshift for the N-CDs, attributing to the different directions and degrees of energy band bending from the interior to the exterior surface, induced by $\mathrm{N}$ radicals, ${ }^{18}$ which probably contributed to the redshift of aptamer-CDs obtained herein. Because of the redshift, the maximum fluorescence emission wavelength of $450 \mathrm{~nm}$ was used in all subsequent experiments.

On using the synthesized aptamer-CDs probes, $K_{\mathrm{d}}$ values of the three aptamer candidates could be determined. As shown in Table 1, the $K_{\mathrm{d}}$ values for the aptamer candidates of PA1, PA2, and PA4 were $15.16 \pm 3.62 \mathrm{nM}, 14.11 \pm 2.59 \mathrm{nM}$, and $16.39 \pm$ $8.14 \mathrm{nM}$ (Fig. S4 $\dagger$ ), respectively, whereas those of PA3 and PA5 were both above $30 \mathrm{nM}$ (Fig. S4 $\dagger$ ). Higher $K_{\mathrm{d}}$ values of aptamer candidates indicated weakened binding affinity to $P$. aeruginosa ${ }^{10,19}$ hence, PA3 and PA5 were excluded from among the aptamer candidates. However, it was still difficult to determine which of the remaining three aptamer candidates (PA1, PA2, and PA4) had the highest specificity to $P$. aeruginosa and the lowest specificity to other interfering bacteria in accordance with their similar $K_{\mathrm{d}}$ values. To resolve this issue, three aptamerCDs/GO systems using aptamer candidates of PA1, PA2, and PA4 were generated to implement the fluorescence assay. ${ }^{19-22}$

\subsection{Specificity of aptamer candidates}

The working principle of using aptamer-CDs/GO system for sensing and detecting $P$. aeruginosa was concurrent with that reported previously. ${ }^{22,23}$ As shown in Fig. 1, in the absence of GO, the fluorescence of aptamer-CDs was prominent (Fig. 1, blue curve). However, when they were mixed together, the aptamer-CDs were absorbed onto the surface of GO via $\pi-\pi$ stacking interactions and the fluorescence of aptamer-CDs was quenched by GO through FRET from aptamer-CDs to GO. ${ }^{22,24}$ Thus, the background signal was reduced to minimum (Fig. 1, black curve). After $P$. aeruginosa was co-incubated with the aptamer-CDs/GO system, the aptamer-CDs would preferentially bind $P$. aeruginos $a$ and the $\pi-\pi$ stacking interactions between the aptamer-CDs and GO were interrupted. Thus, the aptamerCDs were released from the GO sheet and fluorescence was significantly recovered (Fig. 1, red curve).

The working principle above facilitated the determination of the specificities of aptamer candidates. As illustrated in Fig. 2, when $P$. aeruginosa was subjected to the PA1-CDs/GO, PA2-CDs/ GO, and PA4-CDs/GO systems, respectively, all fluorescence recovery ratios exceeded $30 \%$. However, when six interfering 


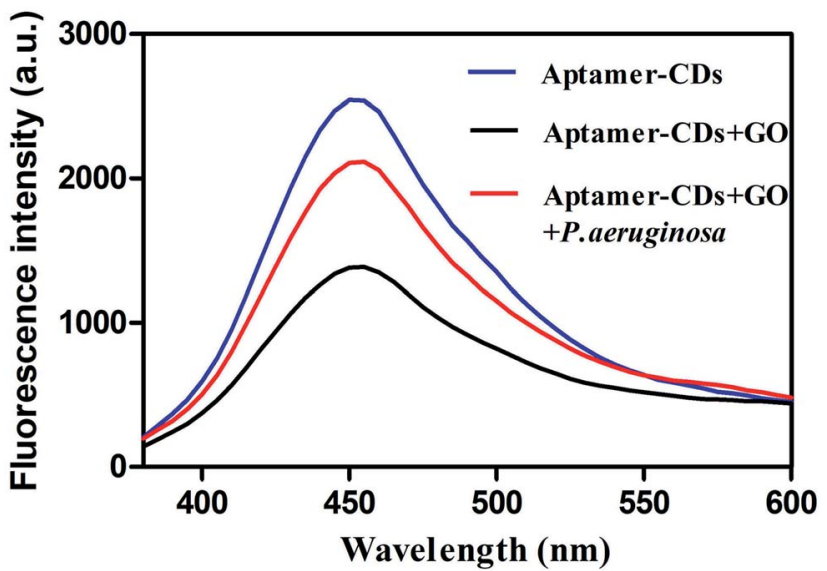

Fig. 1 Working principle of the fluorescence assay to detect $P$. aeruginosa using aptamer-CDs/GO system.

bacteria, namely Pseudomonas putida, Enterococcus faecalis, Clostridium perfringens, E. coli, B. subtilis, and S. aureus, were subjected to these three aptamer-CDs/GO systems, fluorescence recovery ratios were less than $10 \%$. Notably, only the PA1-CDs/ GO system exhibited significantly lower $(p<0.05)$ fluorescence recovery ratios against every interfering bacterium (Fig. 2). Hence, aptamer candidate of PA1 had the highest specificity to the cells of $P$. aeruginosa and the lowest specificity to the cells of every interfering bacteria. Therefore, the PA1 was considered the most appropriate aptamer, with which the highly specific aptamer-CDs/GO system could be generated. This system facilitated the primary fluorescence assay in detecting $P$.

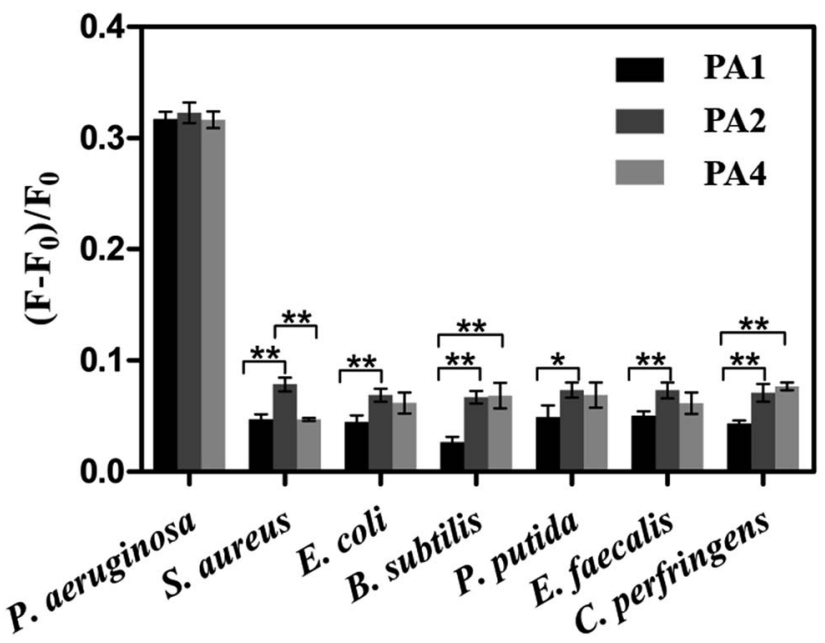

\section{Bacterial strains}

Fig. 2 Specificity of three candidate aptamer-CDs/GO systems towards P. aeruginosa and against Pseudomonas putida, Enterococcus faecalis, Clostridium perfringens, E. coli, B. subtilis, and $S$. aureus. * $(P<0.05), * *(P<0.01)$ : significant difference between two groups. $F=$ fluorescence intensities excited from the aptamer-CDs/ $\mathrm{GO}$ system in the presence of bacterial cells. $F_{0}=$ the fluorescence intensities excited from the aptamer-CDs/GO system in the absence of bacterial cells. aeruginosa (this aptamer-CDs/GO system could only identify the species of $P$. aeruginosa, but could not distinguish the different serotypes, Fig. S6†). However, more experimental conditions should be optimized to further improve this assay.

\subsection{Optimization of reacting conditions}

The fluorescence assay included five parameters, namely reaction $\mathrm{pH}$, reaction temperature, aptamer-CDs probe concentration, GO concentration, and reacting time.

The optimized concentration of the same aptamer-CDs probe was $15 \mu \mathrm{M}$, as reported previously. ${ }^{8}$ Furthermore, the CDs generated herein were $\mathrm{pH}$-sensitive and their fluorescence intensity were stabilized at $\mathrm{pH} 7.5$ after optimization. ${ }^{8}$ Hence, the conditions of $15 \mu \mathrm{M}$ of PA1-CDs probe and $\mathrm{pH} 7.5$ were followed here. Moreover, since the optimal growth temperature for $P$. aeruginosa is $37{ }^{\circ} \mathrm{C}$, the reaction temperature of this aptamer-based assay was set to $37^{\circ} \mathrm{C}$. However, owing to the fluorescence of CDs being quenched by nano-graphite (NG) rather than GO in the previous study, ${ }^{8}$ the optimized concentration of GO for quenching the fluorescence of PA1-CDs probe warranted determination when using $15 \mu \mathrm{M}$ of PA1-CDs probe at $\mathrm{pH} 7.5$ and $37^{\circ} \mathrm{C}$. Fig. 3 shows that fluorescence intensity of PA1-CDs probe decreased with an increase in GO concentration from 0 to $150 \mu \mathrm{g} \mathrm{mL}{ }^{-1}$. However, no increase was observed when GO concentration was greater than $150 \mu \mathrm{g} \mathrm{mL}{ }^{-1}$. Therefore, $150 \mu \mathrm{g} \mathrm{mL}^{-1}$ was determined as the optimal GO concentration, this value being similar to that of NG for quenching the fluorescence of the same CDs. ${ }^{8}$

Finally, the reacting time for the incubation of the cells of $P$. aeruginosa with PA1-CDs/GO system was optimized under the previously optimized conditions. Fig. S5† showed that the fluorescence recovery ratio peaked at $60 \mathrm{~min}$ and did not increase any further after longer incubations; hence, 60 min was the selected as the optimal reaction time. Therefore, the final optimized experimental conditions for the aptamer-CDs/GOmediated assay were as follows: $15 \mu \mathrm{M}$ of aptamer-CDs probe, $150 \mu \mathrm{g} \mathrm{mL}{ }^{-1}$ of $\mathrm{GO}, \mathrm{pH} 7.5,37^{\circ} \mathrm{C}$, and $60 \mathrm{~min}$.

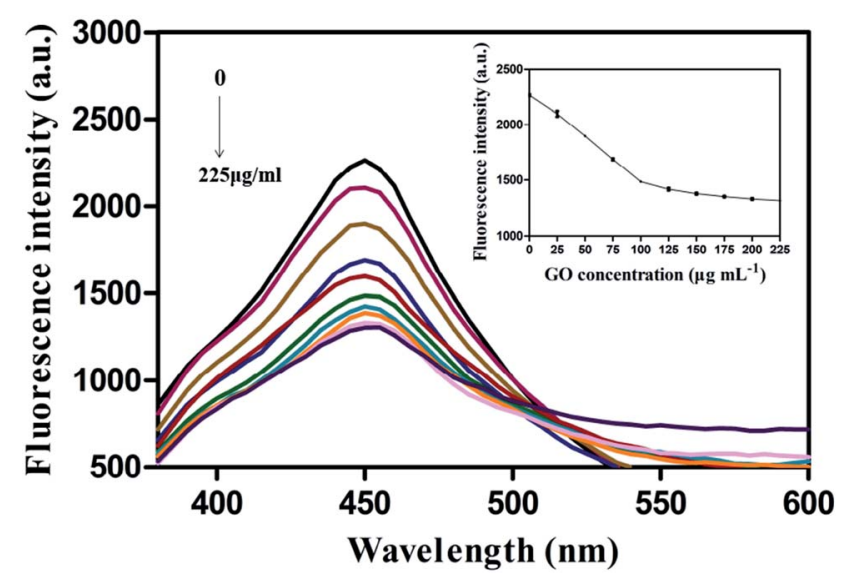

Fig. 3 Optimization of GO concentration for the fluorescence assay based on the PA1-CDs/GO system. 


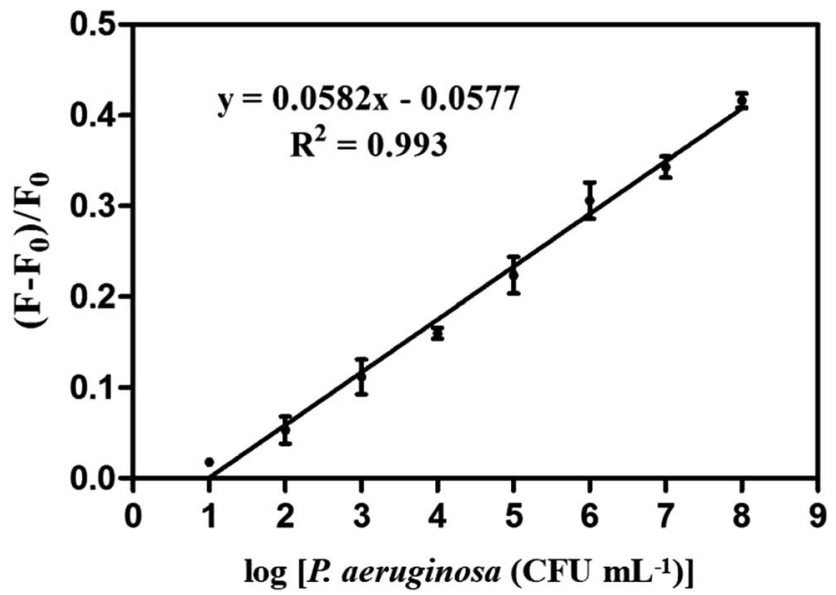

Fig. 4 Linear correlation between the fluorescence recovery ratio and the density of $P$. aeruginosa. $F=$ fluorescence intensities excited from the aptamer-CDs/GO system in the presence of $P$. aeruginosa. $F_{0}=$ the fluorescence intensities excited from the aptamer-CDs/GO system in the absence of $P$. aeruginosa.

\subsection{Analytical application}

Under optimized conditions, fluorescence recovery ratios for the PA1-CDs/GO system with P. aeruginosa at different densities were determined to evaluate the application of this fluorescence assay. As illustrated in Fig. 4, fluorescence recovery ratios exhibited a linear correlation with various densities of $P$. aeruginosa ranged from 10 to $10^{8} \mathrm{CFU} \mathrm{mL}^{-1}$, the calibration curve was expressed by the regression equation of $\left(F-F_{0}\right) / F_{0}=$ $0.0582 \log \left[C\left(\mathrm{CFU} \mathrm{mL} \mathrm{mL}^{-1}\right)\right]-0.057$ with a correlation coefficient of 0.993 ( $F$ and $F_{0}$ represented the fluorescence intensities excited from the aptamer-CDs/GO system in the presence and absence of $P$. aeruginosa, respectively). According to the equa-

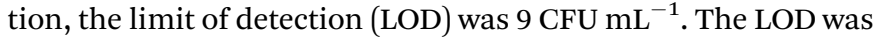
an important parameter reflecting the sensitivity of detection., ${ }^{3,16}$ Previously, an aptamer-based FISH assay was established for detecting $P$. aeruginosa. However, the LOD was unclear. $^{10}$ The presently developed fluorescence assay approached a fine LOD that specified in a previous study on $P$.

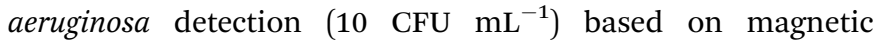
enrichment and nested PCR. ${ }^{1}$ Nevertheless, our aptamer-CDs/ GO mediated detecting assay was less time-consuming, indicating that this assay could achieve rapid and highly sensitive detection of $P$. aeruginosa.

\subsection{Evaluation for real samples of the assay}

Our fluorescence assay was conducted at the optimized conditions as describes above. However, its application for actual samples under the natural conditions also warranted investigation to evaluate the practicality of this assay. This evaluation was completed in simulative samples of tap water and commercial bottled water, which were artificially contaminated by $P$. aeruginosa. Table 2 shows that the cell densities of $P$. aeruginosa experimentally detected using the presently developed fluorescence assay were like those obtained via microscopic enumeration in all the two samples,
Table 2 Detection results of $P$. aeruginosa in real water samples ${ }^{a}$

\begin{tabular}{llll}
\hline & $\begin{array}{l}\text { Microscopic counted } \\
\text { concentration } \\
\text { Samples }\end{array}$ & $\begin{array}{l}\text { Experimentally obtained } \\
\text { concentration } \\
\left(\mathrm{CFU} \mathrm{mL}^{-1}\right)\end{array}$ & RSD (\%) \\
\hline Tap & 1000 & 865 & 2.30 \\
water & 100 & 87 & 3.44 \\
& 50 & 32 & 6.37 \\
Bottled & 1000 & 896 & 1.90 \\
water & 100 & 89 & 3.39 \\
& 50 & 42 & 5.18 \\
${ }^{a}$ RSD: relative standard deviation. & \\
\end{tabular}

thereby indicating the applicability of this assay for water samples to detect potential $P$. aeruginosa contamination.

\section{Conclusions}

In this study, an aptamer-CDs/GO-based fluorescence assay was developed for detecting $P$. aeruginosa. During SELEX, HTS facilitated clear recognition of aptamer candidates with high efficiency. This fluorescence assay exhibits high specificity and sensitivity. Moreover, this assay is easy to operate and comprises environmentally friendly materials. This assay has potential applications with actual water samples with satisfactory accuracy. These characteristics highlight that this assay is potential in the application of detecting the contamination of $P$. aeruginosa for the water quality supervision.

\section{Conflicts of interest}

There are no conflicts to declare.

\section{Acknowledgements}

This study was supported by China Postdoctoral Science Foundation (Grant No. 2016M590657) and National Natural Science Foundation of China (Grant No. 31500029).

\section{Notes and references}

1 Y. J. Tang, Z. Ali, J. Zou, K. Yang, X. B. Mou, Z. Y. Li, Y. Deng, Z. X. Lu, C. Ma, M. A. A. Shah, S. Elingarami, H. W. Yang and N. Y. He, J. Nanosci. Nanotechnol., 2014, 14, 4886-4890.

2 P. Deschaght, S. Van daele, F. De Baets and M. Vaneechoutte, J. Cystic Fibrosis, 2011, 10, 293-297.

3 N. Alizadeh, M. Y. Memar, S. R. Moaddab and H. S. Kafil, Biomed. Pharmacother., 2017, 93, 737-745.

4 M. R. Dunn, R. M. Jimenez and J. C. Chaput, Nat. Rev. Chem., 2017, 1, 0076.

5 N. Nguyen Quang, G. Perret and F. Duconge, Pharmaceuticals, 2016, 9, 76.

6 Y. S. Kim, N. H. A. Raston and M. B. Gu, Biosens. Bioelectron., 2016, 76, 2-19.

7 P. Namdari, B. Negahdari and A. Eatemadi, Biomed. Pharmacother., 2017, 87, 209-222. 
8 L. Zhu, G. H. Xu, Q. Song, T. Tang, X. Wang, F. D. Wei and Q. Hu, Sens. Actuators, B, 2016, 231, 506-512.

9 M. Joo, S. H. Baek, S. A. Cheon, H. S. Chun, S. W. Choi and T. J. Park, Colloids Surf., B, 2017, 154, 27-32.

10 K. Y. Wang, Y. L. Zeng, X. Y. Yang, W. B. Li and X. P. Lan, Eur. J. Clin. Microbiol. Infect. Dis., 2011, 30, 273-278.

11 A. G. Souza, K. Marangoni, P. T. Fujimura, P. T. Alves, M. J. Silva, V. A. F. Bastos, L. R. Goulart and V. A. Goulart, Exp. Cell Res., 2016, 341, 147-156.

12 N. Duan, X. Y. Ding, S. J. Wu, Y. Xia, X. Y. Ma, Z. P. Wang and J. Chen, J. Microbiol. Methods, 2013, 94, 170-174.

13 M. Zuker, Nucleic Acids Res., 2003, 31, 3406-3415.

14 S. J. Zhu, Q. N. Meng, L. Wang, J. H. Zhang, Y. B. Song, H. Jin, K. Zhang, H. C. Sun, H. Y. Wang and B. Yang, Angew. Chem., Int. Ed., 2013, 52, 3953-3957.

15 S. H. Suh, H. P. Dwivedi, S. J. Choi and L. A. Jaykus, Anal. Biochem., 2014, 459, 39-45.

16 A. Abbaspour, F. Norouz-Sarvestani, A. Noon and N. Soltani, Biosens. Bioelectron., 2015, 68, 149-155.
17 J. Hoinka, A. Berezhnoy, P. Dao, Z. E. Sauna, E. Gilboa and T. M. Przytycka, Nucleic Acids Res., 2015, 43, 5699-5707.

18 S. L. Hu, R. X. Tian, Y. G. Dong, J. L. Yang, J. Liu and Q. Chang, Nanoscale, 2013, 5, 11665-11671.

19 S. H. Lee, J. Y. Ahn, K. A. Lee, H. J. Um, S. S. Sekhon, T. S. Park, J. Min and Y. H. Kim, Biosens. Bioelectron., 2015, 68, 272-280.

20 J. Teng, F. Yuan, Y. Ye, L. Zheng, L. Yao, F. Xue, W. Chen and B. Li, Front Microbiol., 2016, 7, 1426.

21 M. Shandordizadeh, S. M. Taghdisi, N. Ansari, F. A. Langroodi, K. Abnous and M. Ramezani, Sens. Actuators, B, 2017, 241, 619-635.

22 Z. P. Liu and X. G. Su, Biosens. Bioelectron., 2017, 87, 66-72. 23 Z. M. Zhou, J. Zhou, J. Chen, R. N. Yu, M. Z. Zhang, J. T. Song and Y. D. Zhao, Biosens. Bioelectron., 2014, 59, 397-403.

24 Y. J. Guo, L. Deng, J. Li, S. J. Guo, E. K. Wang and S. J. Dong, ACS Nano, 2011, 5, 1282-1290.

25 Y. Lian, F. J. He, H. Wang and F. F. Tong, Biosens. Bioelectron., 2015, 65, 314-319. 